\title{
Sweet's Syndrome in Association with Ulcerative Colitis and Dyserythropoietic Anemia
}

\author{
Mahmud Natour $^{\mathrm{a}}$ Yehuda Chowers $^{\mathrm{b}}$ Michal Solomon ${ }^{\mathrm{c}}$ Marat Khaikin $^{\mathrm{a}} \quad$ Iris Barshack $^{\mathrm{d}}$ \\ Amram Ayalon $^{\mathrm{a}}$ Oded Zmora $^{\mathrm{a}}$
}

Departments of a Surgery and Transplantation, ${ }^{b}$ Gastroenterology, ${ }^{c}$ Dermatology and ${ }^{d}$ Pathology, Sheba Medical Center, Sackler School of Medicine, Tel Aviv University, Tel Aviv, Israel

Dear Sir,

Sweet's syndrome is a rare autoimmune condition which manifests as red or brown painful skin lesions, most commonly in the upper extremities, face and neck. The mainstay of treatment involves high doses of systemic steroids, usually resulting in rapid resolution of symptoms [1]. Rare cases of Crohn's disease and ulcerative colitis associated with Sweet's syndrome have been reported in the literature [2].

This letter reports a nontypical presentation of inflammatory bowel disease (IBD), most probably ulcerative colitis, wherein Sweet's syndrome as well as severe anemia preceded an unusual intestinal manifestation of IBD.

\section{Case Presentation}

A 45-year-old female was admitted to the hospital for weakness, weight loss and general deterioration; the patient did not complain of abdominal pain or other gastrointestinal symptoms. Two months earlier, she presented with severe anemia, high-grade fever, arthralgia and multiple skin lesions over her extremities. The lesions were well demarcated, erythematous and edematous papules and plaques, warm and tender to palpation (fig. 1). A skin biopsy specimen obtained from a plaque revealed edema and a dense neutrophilic infiltrate of the papillary dermis without leukocytoklastic vasculitis, compatible with the diagnosis of Sweet's syndrome. She was treated with systemic steroids with rapid symptomatic improvement. The steroid dose was gradually tapered.

Her medical history was significant for anemia for 7 years, worsening in conjunction with any concomitant disease, and occasionally requiring blood transfusions. Based on clinical features and bone marrow aspiration, hematologic work up suggested the diagnosis of congenital dyserythropoietic anemia.

The patient developed diffuse abdominal pain the day following her admission. Abdominal CT scan indicated severe pancolitis and free fluid within the abdominal cavity. Conservative treatment with broadspectrum antibiotics, intravenous hydration, and bowel rest was initiated. However, within $48 \mathrm{~h}$ she developed worsening abdominal pain, signs of peritoneal irritation, tachypnea, and tachycardia. The patient was taken to the operating room emergently and the entire colon was found to be severely inflamed and thickened with 2 microperforations. The small bowel appeared normal throughout its length. Total abdominal colectomy with Hartmann's pouch, which was closed at the upper rectum, and an ileostomy were performed.

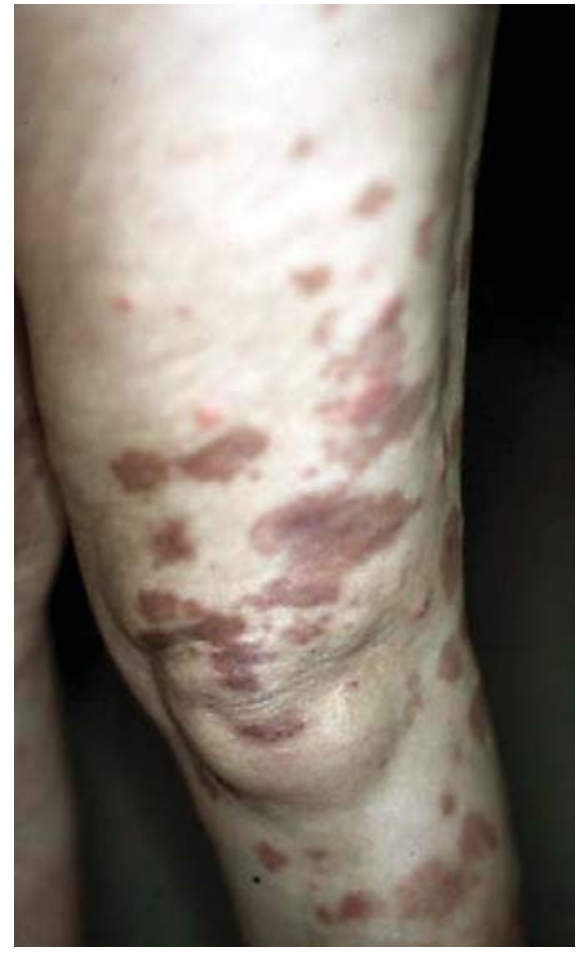

Fig. 1. Maculopapular rash of the lower extremity, which was diagnosed as Sweet's syndrome 2 months prior to the presentation of intestinal disease.

\section{KARGER}

Fax +41 613061234

E-Mail karger@karger.ch

www.karger.com
(C) 2007 S. Karger AG, Basel

0012-2823/07/0753-0142\$23.50/0

Accessible online at:

www.karger.com/dig
Oded Zmora, MD

Department of Surgery and Transplantation, Sheba Medical Center

IL-52621 Tel Hashomer (Israel)

Tel. +972 3530 2247, Fax +972 3534 1097, E-Mail ozmora@post.tau.ac.il 
Pathologic examination of the colon confirmed severe acute IBD with features of indeterminate colitis. Morphologic features such as diffuse involvement of the colon and absence of ileal disease or granulomas were highly suggestive for ulcerative colitis.

Postoperatively, the patient was gradually weaned off steroidal therapy and remained asymptomatic without any gastrointestinal complains, skin lesions, or hematologic disorders. Repeated blood counts showed normal hemoglobin levels.

\section{Discussion}

Sweet's syndrome, also known as acute febrile neutrophilic dermatoses, was first described in 1964 by Robert Douglas Sweet. Fever, tender raised erythematous plaques or nodules, leukocytosis, an elevated erythrocytes sedimentation rate, and a predominantly neutrophilic infiltration in the dermis without vasculitis characterize the syndrome [3]. Typically, there is an excellent response to treatment with systemic corticosteroids. Classical or idiopathic Sweet's syndrome, which may follow an upper respiratory or gastrointestinal tract infection, is the most common type, occurring in $70 \%$ of the cases. Other associated conditions include neoplasia, mostly hematological malignancy, various inflammatory diseases, drug-induced Sweet's syndrome and pregnancy [4-6].
The association of Sweet's syndrome with IBD has rarely been reported. Although Sweet's syndrome has reportedly been associated more frequently with Crohn's disease [2], cases of ulcerative colitis have also been reported [7]. Interestingly, in all cases in which IBD was associated with Sweet's syndrome, significant colitis was noted.

Histological examination of the cutaneous plaques in Sweet's syndrome shows severe neutrophilic infiltration of the dermis without vasculitis or panniculitis. It is important to note that part of the histologic features of the cutaneous lesions in Sweet's syndrome may have some parallel features to erythema nodosum associated with IBD, and the sound combination of clinical assessment and histopathology is essential for the correct diagnosis.

In this case presentation, the manifestations of Sweet's syndrome preceded the eruption of intestinal IBD; the skin lesions rapidly responded to steroids which is typical of Sweet's syndrome. However, an attempt to wean the patient off steroids resulted in an atypical aggressive episode of fulminant colitis; this indicated a diagnosis of IBD, highly suggestive of ulcerative colitis.

Interestingly, this patient also had a 7year history of chronic anemia, which completely resolved after total abdominal colectomy. Although she had never undergone bone marrow biopsy, clinical features were suggestive of a diagnosis of congenital dyserythropoietic anemia. Regardless of whether the diagnosis of congenital dyserythropoietic anemia was correct, the appearance of anemia 7 years prior to the intestinal manifestations of IBD, which completely resolved with total abdominal colectomy, is highly atypical.

\section{References}

1 Cohen PR, Kurzrock R: Sweet's syndrome revisited: a review of disease concepts. Intern J Dermatol 2003;42:761-778.

2 Rappaport A, Shaked M, Landau M, Dolev E: Sweet's syndrome in association with Crohn's disease: report of a case and review of the literature. Dis Colon Rectum 2001;44:15261529.

3 Von den Driesch P: Sweet's syndrome (acute febrile neutrophilic dermatosis). J Am Acad Dermatol 1994;31:535-536.

4 Naz E, Ruano M, Vidaurrazage C, Cassado M, Fernandez-Jimenez MC, Bustos JG, Hernandez-Navaro F: Sweet's syndrome as a lifethreatening dermatosis. Am J Med 2000;109: 73-74.

5 Walker DC, Cohen PR: Trimethoprim-sulfamethoxazole associated with acute febrile neutrophilic dermatosis: a case report and review of drug-induced Sweet's syndrome. J Am Acad Dermatol 1996;34:918-923.

6 Cohen PR: Pregnancy-associated Sweet's syndrome: world literature review. Obstet Gynecol Surv 1993;48:584-587.

7 Diaz-Peromingo JA, Garcia-Suarez F, Sanchez-Liera J, Saborido-Frojan J: Sweet's syndrome in a patient with acute ulcerative colitis: presentation of a case and review of literature. Yale J Biol Med 2001;74:165-168. 